\title{
Local Effects during Indentation of Fully Supported Sandwich Panels with Micro Lattice Cores
}

\author{
R.A.W.Mines ${ }^{a}$, S.McKown, S.Tsopanos, E.Shen, W.Cantwell, W.Brooks, \\ C.Sutcliffe
}

Department of Engineering, University of Liverpool, Brownlow Street, Liverpool, L69 3GH, UK

ar.mines@liv.ac.uk

Keywords: micro-lattice, cellular, sandwich, panel, indentation

\begin{abstract}
This paper discusses the penetration behavior of fully supported sandwich panels with micro-lattice and foam cores, and composite skins. This behaviour is of importance during foreign object impact and perforation of sandwich structures. Experimental results are given for quasi-static penetration of micro-lattice and foam blocks, and it is shown that these two cellular materials are comparable. Experimental results are also given for drop weight penetration of fully supported skinned panels, and it is shown that skin failure and core penetration are also similar for the two core materials. It is concluded that there is scope for improving the performance of micro-lattice structure and so making such material superior to that of aluminium foam.
\end{abstract}

\section{Introduction}

Mines [1] discusses the new manufacturing technique of selective laser melting to realize stainless steel micro lattice structures, which have strut diameters of 200 microns and strut lengths of $3 \mathrm{~mm}$. In the manufacturing process a bed of fine powder (15 to 30 microns) is selectively melted using a laser. A new layer of powder is deposited, and the process is repeated. In this way, cellular structures can be built in 150 micron layers. Fig. 1(a) shows some blocks, after the powder has been cleaned away. Note the steel base plate. Fig. 1(b) gives the unit cell, which we call body centred cubic (bcc). It should be noted that each laser pass takes about 30 seconds, which gives a total build time of about 6 hours.
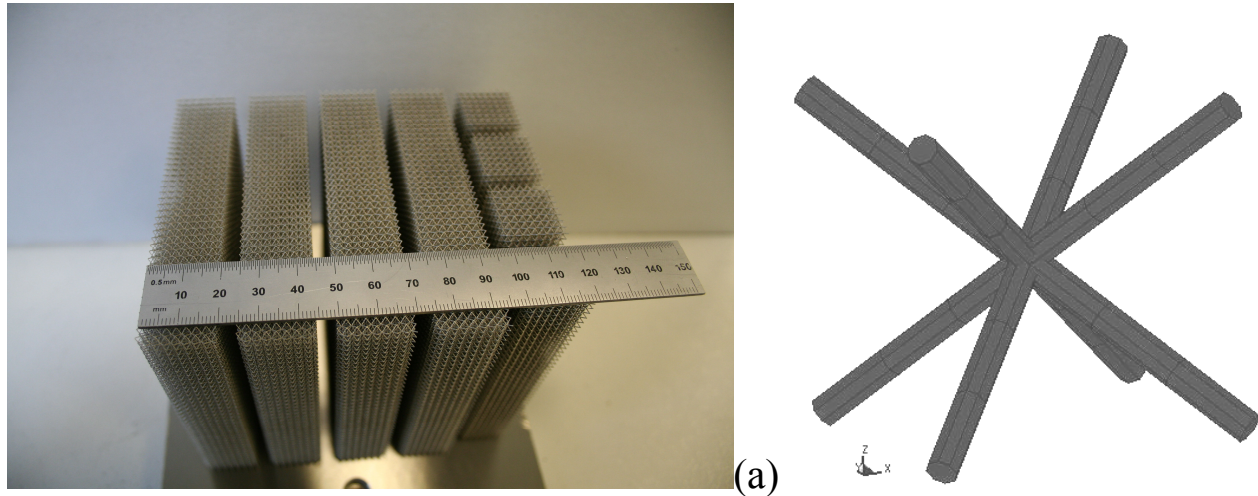

Figure 1 : (a) BCC cellular structures for panel $(100 \mathrm{~mm} \times 100 \mathrm{~mm} \times 20 \mathrm{~mm})$ and beam $(100 \mathrm{~mm} \times$ $20 \mathrm{~mm} \times 20 \mathrm{~mm}$ ) and (b) body centred cubic (bcc) unit cell

There is interest in using these micro-lattice structures as core material in sandwich structures, to replace such cellular materials as aluminium honeycomb or aluminium foam. The specific motivation for the application of this technology is in designing mitigation against foreign object impact damage in sandwich panels, e.g. hail or rubber impact on twin skinned aircraft fuselages.

Mines [1] has identified five behaviours for perforation of a sandwich panel, namely: global elastic response, local core skin debonding, perforation core crushing, boundary response and post 
impact response. This paper focuses on perforation core crushing. The paper discusses the penetration of the core on its own under static loading, and with a composite skin under drop weight loading. Results are compared with Alporas aluminium foam [2].

\section{Response of core only (quasi static)}

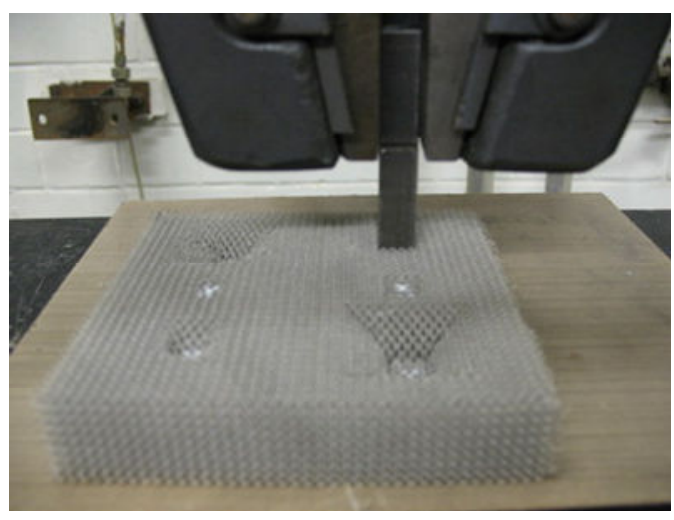

BCC lattice panel

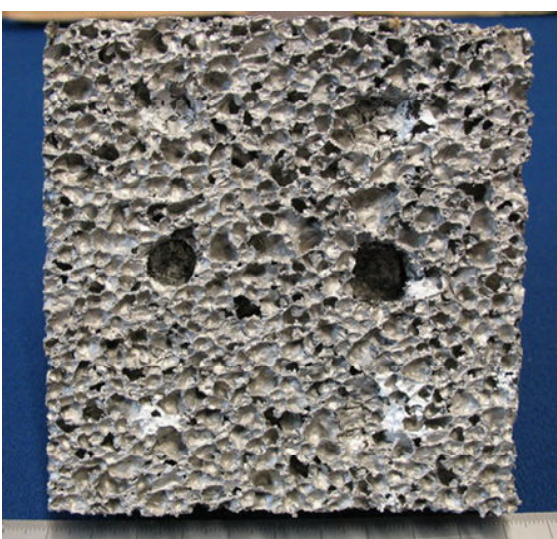

Aluminium foam panel

Figure 2: Penetration of micro- lattice and foam

Fig. 2 shows some static penetration tests, in which a $50 \mathrm{kN}$ servo hydraulic machine was used to drive either a $10 \mathrm{~mm}$ square and hemispherical hard steel object into the material. The displacement rate was $2 \mathrm{~mm}$ per minute. Force was measured by a load cell and displacement was measured from the crosshead. The photos show $100 \mathrm{~mm} \times 100 \mathrm{~mm}$ by $20 \mathrm{~mm}$ blocks being indented at three different displacements, with hemispherical indenter on the left and square indenter on the right of each photo. Fig. 3 shows relevant force displacement plots and Fig. 4 gives some cross-sections, both for the spherical indenter There are three plots shown on each graph, relating to the three energies. For the cross section photos, the tested blocks were sectioned with a diamond circular saw with a view to minimise damage from cutting.

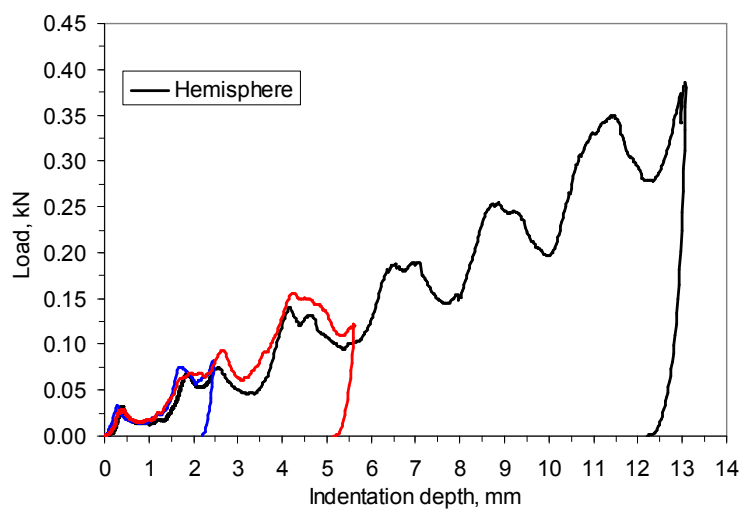

(a) BCC panel indentation (hemisphere).

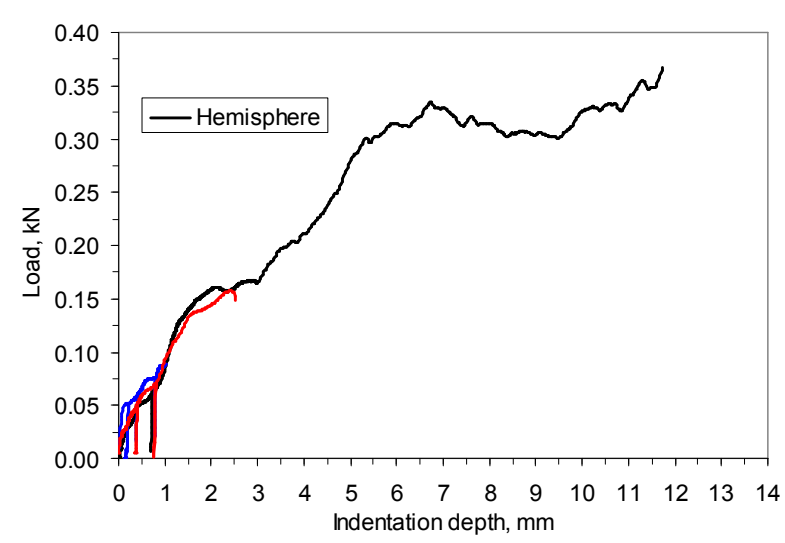

(b) $\mathrm{Al}$ foam panel indentation (hemisphere).

Figure 3: Indentation for bcc (left) and foam (right) 

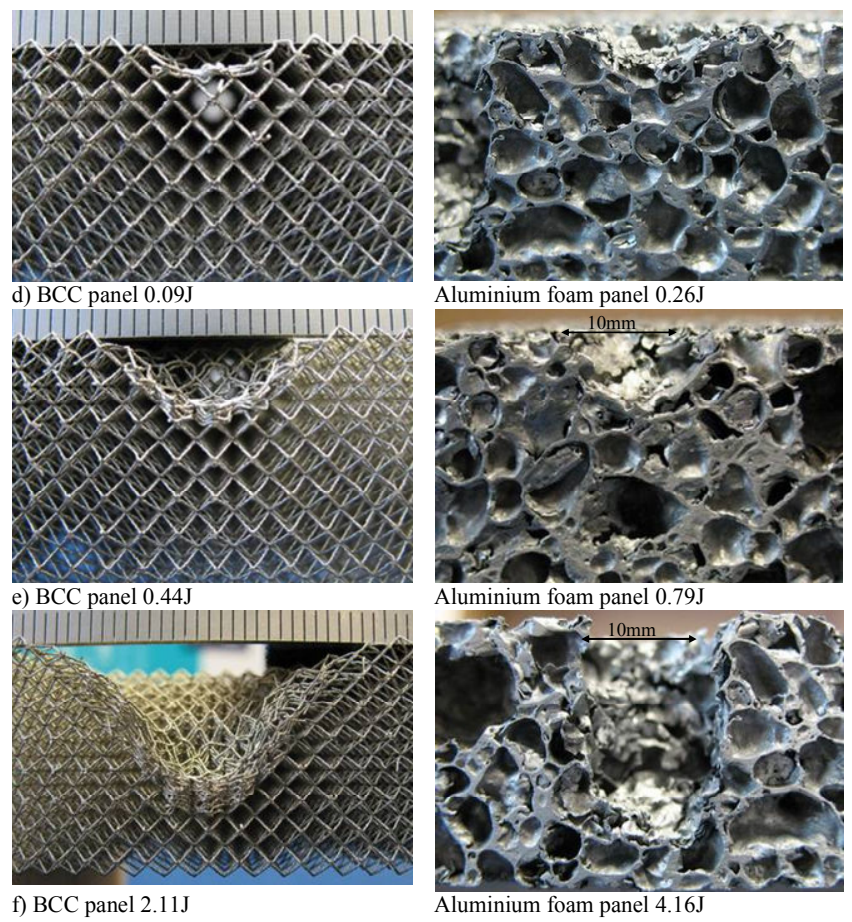

Figure 4: Cross-sections at various penetration energies.

From the graphs, it can be seen that the force for the micro-lattice is steadily rising, with oscillations associated with tensile stretching of the surface of the micro lattice (see the cross-sections). On the other hand, in the case of the aluminium foam, the micro mechanics of penetration is dominated by shearing of the foam adjacent to the indenter, and crushing of the foam under the indenter. The crush characteristic for the foam is less oscillatory, but has a similar rising curve with penetration. It can be concluded that the micro mechanics of penetration are completely different for the two micro structures. It can also be concluded that the square indenter gives higher penetration loads for both cellular structures, and that the foam gives higher loads as compared with the micro-lattice. The density of the micro lattice and foams are similar, 250 and $300 \mathrm{~kg} / \mathrm{m} 3$ respectively, which means that the specific energy absorption is similar. It should be noted that in the future micro lattice structure will be manufactured using titanium powder. This may lead to better specific properties for the lattice, but the ductility of such titanium micro-lattices may be less than the stainless steel case. Fig. 5 summarises core only results, and shows the effects of core type and indenter type.

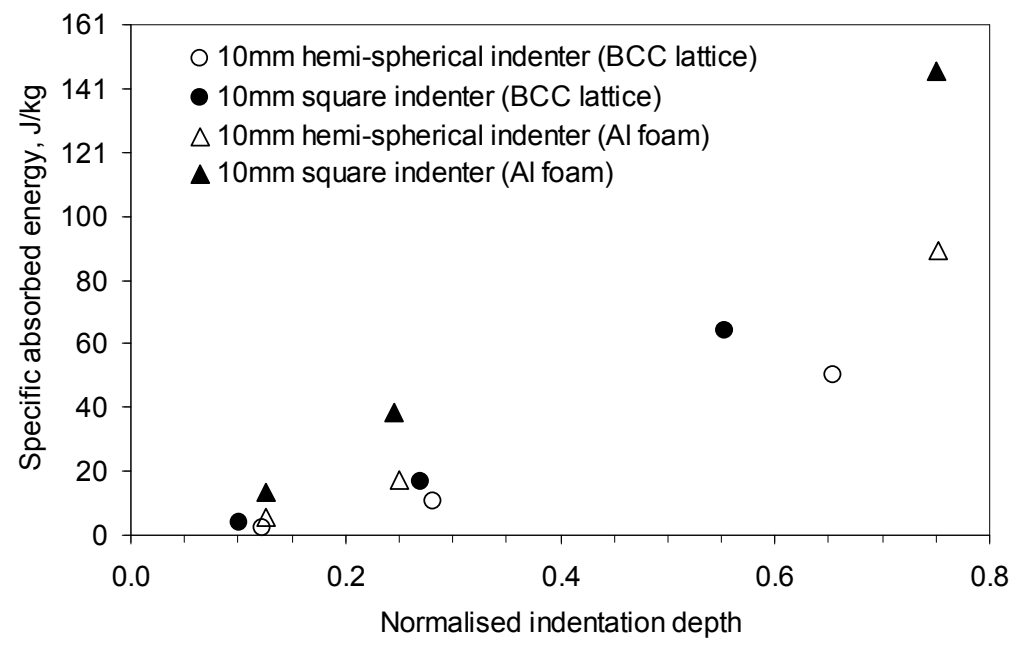

Figure 5: Summary of core only results 


\section{Core with skin (low velocity impact)}

The next series of tests concerned the penetration of the core with 4 ply carbon epoxy skins. In this case, drop weight loading was considered, with impact velocities up to $3 \mathrm{~m} / \mathrm{s}$ (drop height $3 \mathrm{~m}$ ). Hence, strain rate and inertial effects will occur. However, it is assumed here that these are of second order, and so we can compare static skinless results with impact skinned results. A simple drop tower was used, and force was measured with a load cell attached to the impactor and displacement was derived from a Laser Doppler Velocimeter. The same boundary condition as the static skinless case was considered, namely full support with hemispherical impactor. Fig. 6 shows the fully supported configurations for the lattice and aluminium foam panels. Nine impact energies were used for each panel, in order to obtain various amounts of penetration.

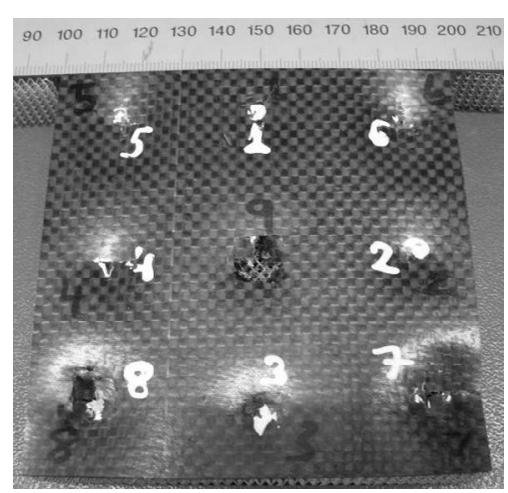

(a) Lattice panel bcc\#2

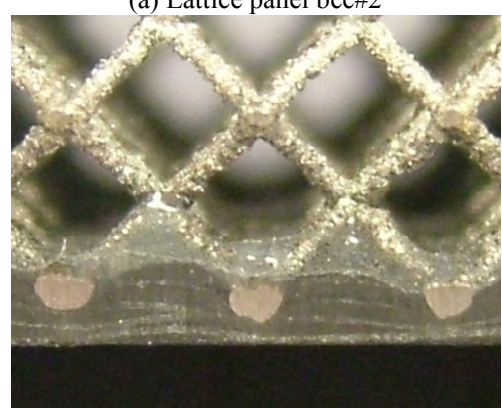

(c) Lattice-skin interface panel bcc\#2

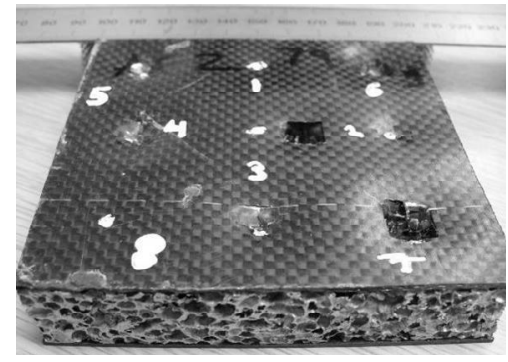

(b) Aluminium foam panel ALF

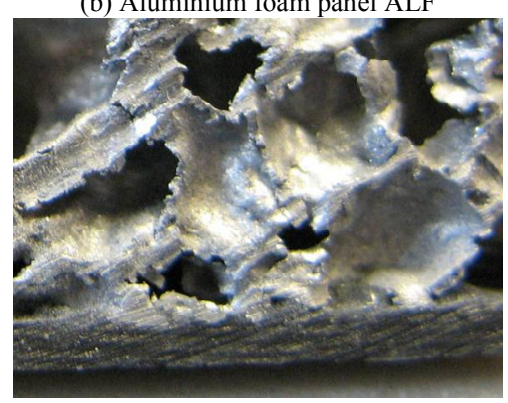

(d) Aluminium foam-skin interface panel ALF

Figure 6: Penetrated micro lattice (left) and foam (right panels)

A major issue in this work is core skin bond strength. Fig. 6 gives details of this. In both cases, the skins were hot pressed onto the panels, which means that the cores were pushed into the skins. This was especially true for the micro-lattice, which means that good core skin adhesion was found. However, the strength of these core-skin bonds need to be quantified. Fig. 7 gives some crosssections after impact and Fig. 8 gives load v displacement data 


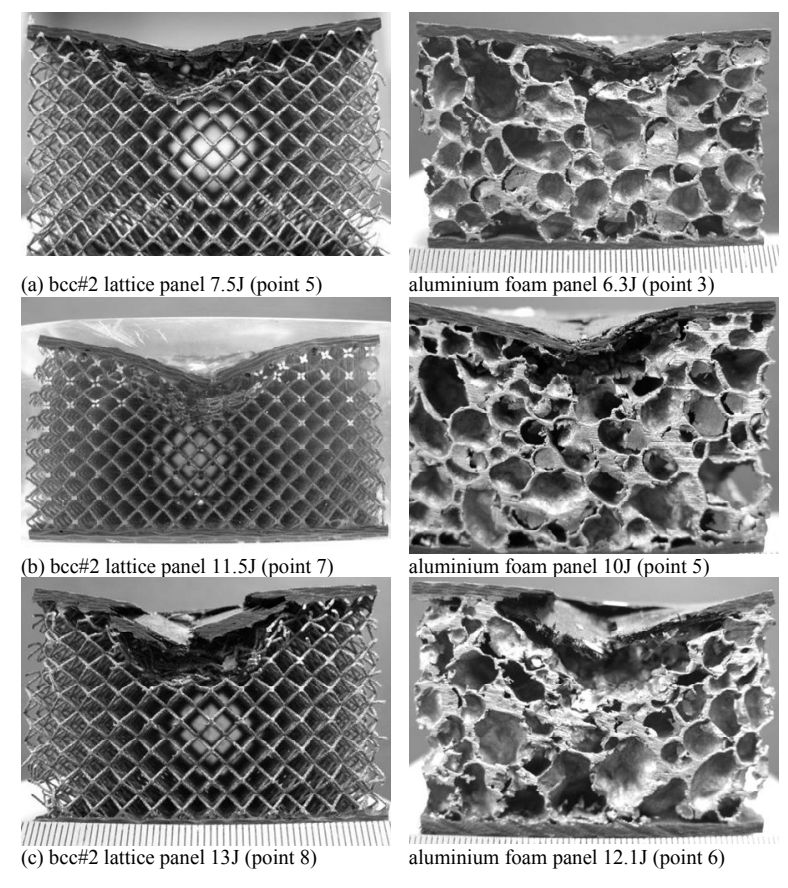

Figure 7: Cross-sections at various impact energies

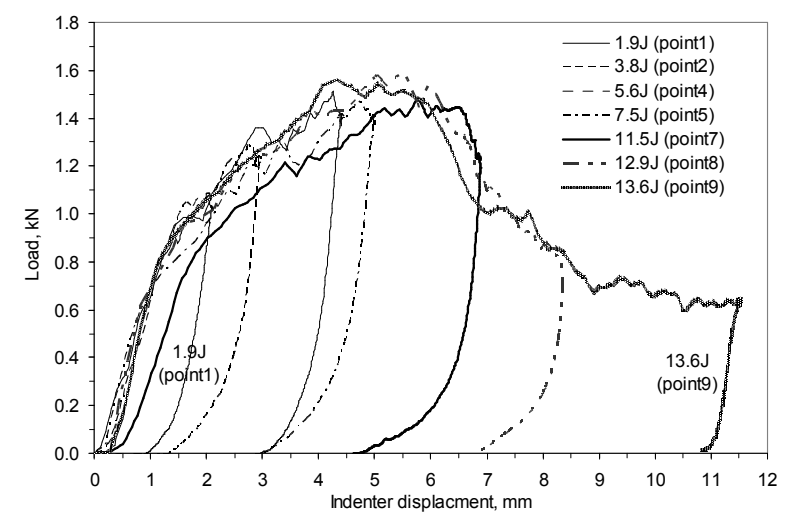

(a) bcc\#2 lattice panel (mass 107g)

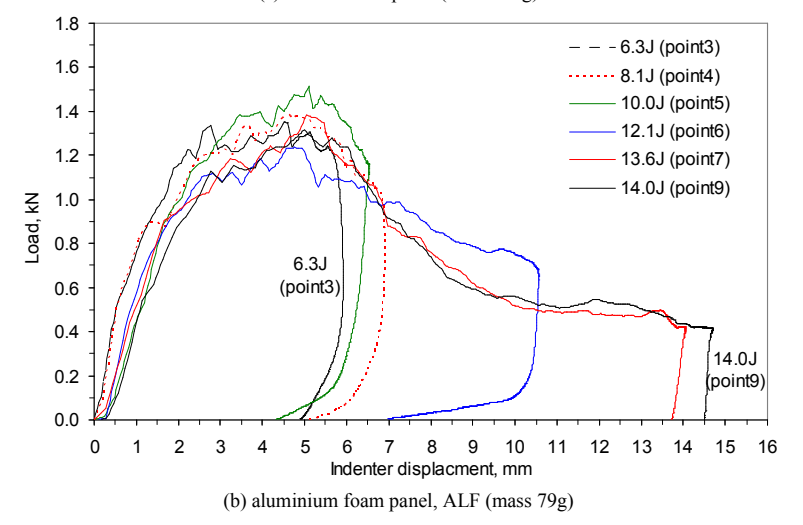

Figure 8: Load v indenter displacement results

The cross-section data shows very similar damage for the micro lattice and aluminium foam. The failure modes of the skins are similar, suggesting that the progressive degradation of skin support leading to skin rupture is similar. As far as the load v displacement data is concerned, there is little change with impact energy. The loss of linearity relates to skin damage and the post maximum load relates to full perforation. 


\section{General Discussion and Concluding Remarks}

This work is a preliminary investigation relating to the foreign object impact of sandwich panels with micro-lattice cores. Although much work in the literature concerns the deformation of blocks of core material, where various constitutive laws can be developed and calibrated, there is little work on the penetration of cellular materials, where intense shear effects occur. This has been commented upon by Hanssen et al [3].

It has been shown that the existing micro-lattice (Stainless Steel $316 \mathrm{~L}$ and BCC) is comparable with Alporas aluminium foam for perforation behaviour. This suggests that there is scope to improve the specific perforation performance of the micro-lattice by changing the micro structure or the parent material (i.e. to titanium).

This data is being used to validate and calibrate a numerical simulation of the progressive collapse of micro-lattice structures, using DYNA. Each strut is modeled using three Hughes Liu beam elements. In the longer term, other micro-lattice geometries [1] will be studied with a view to improving perforation performance.

\section{Acknowledgements}

Support from grants sponsored by EPSRC/EP/C009525/1, EPSRC/EP/009398/1 and EU FP6 CELPACT is gratefully acknowledged.

\section{References}

[1] R.Mines, Strain, Vol. 44, pp71-83, (2008)

[2] S. McKown, R.A.W Mines, Appl. Mech. Mater. Vol. 1-2, pp211-216 (2004)

[3] A.G. Hanssen, O.S. Hopperstad, M. Langseth, H. Ilstad, Int. J. Mech. Sci. Vol. 44, pp359406, (2002) 